\title{
Suspected lung cancer: its initial management and staging
}

\section{Daniel K C Lee*}

\author{
Department of Thoracic Oncology, Papworth Hospital, Papworth Everard, Cambridge, CB3 8RE, UK
}

Received 12th January 2006; accepted 16th December 2006

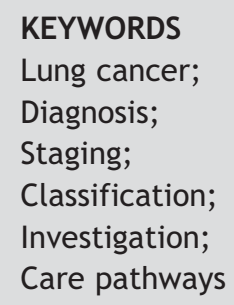

\begin{abstract}
Lung cancer has traditionally been viewed as being difficult to treat and generally associated with a poor outcome. With new advances in technology and medicine in general, the public has come to expect a better prognosis from lung cancer. Once a patient has been referred with suspected lung cancer, it is important to confirm the diagnosis and to stage the cancer; in this way, one can ascertain whether or not the cancer is potentially operable. By providing definitive surgery, the aim is to cure the patient of cancer. The role of staging extends beyond that of merely directing appropriate therapy or determining the patient pathway (be it supportive, palliative or curative); it also has prognostic implications including the likelihood of survival from lung cancer.

(c) 2007 General Practice Airways Group. All rights reserved.
\end{abstract}

\section{Introduction}

The incidence of lung cancer is on the increase globally and the greatest rise over the past decade has been observed in women. This has been directly attributed to women taking up smoking in the quest for social equality with men. Lung cancer has traditionally been viewed as being difficult to treat and is generally associated with a poor outcome. However, progress is being made, with ongoing research looking at different therapies in addition to different therapeutic approaches and options. With new advances in technology and medicine in general, the public has come to expect a better prognosis from lung cancer. Given that patients with

* Corresponding author.

Tel: +44 (0)1480 830541; fax: +44 (0)1480 831315

E-mail address: dkclee@doctors.org.uk suspected lung cancer will usually present to their general practitioner (GP) first, the role of the GP is therefore pivotal in the management of these patients.

It is little appreciated that for patients to receive optimal therapy, they must first be correctly staged for their lung cancer. In its crudest form, staging for lung cancer entails finding out how advanced the cancer is in terms of its local and distant effects. It is important to ascertain whether a particular lung cancer is potentially operable, since by undergoing definitive surgery there is a likelihood of cure. However, the role of staging extends beyond that of merely directing appropriate therapy or determining the patient pathway - be it supportive, palliative or curative - since it also carries with it prognostic implications including the likelihood of survival. Traditional staging methods embrace invasive surgical procedures that are today still considered the gold

1471-4418 @ 2007 General Practice Airways Group. All rights reserved doi:10.3132/pcrj.2007.00019 


\begin{tabular}{l} 
Box $1 \quad$ Clinical features requiring an urgent CXR \\
\hline Haemoptysis - always requires a CXR \\
Any of the following unexplained symptoms or \\
signs persisting for more than three weeks: \\
Breathlessness \\
Chest signs \\
Cough that is unexplained or persisting \\
Features suggestive of metastases from lung cancer \\
(bone, brain, liver or skin) \\
Finger clubbing \\
Hoarseness of voice \\
Pain in the chest or shoulder \\
Persistent cervical or supraclavicular \\
lymphadenopathy \\
Weight loss \\
\hline
\end{tabular}

standard. There is, however, a strong trend and imminent move towards better lung cancer staging with less invasive techniques in line with recent advances in technology.

This Discussion paper starts with a Case study on the initial primary care management of a patient presenting with suspected lung cancer, and then briefly reviews the approach to staging the cancer once the patient has been referred and a definitive diagnosis has been made.

\section{Case study}

A 60-year-old ex-smoker presents to your surgery with mild breathlessness and cough. These symptoms have persisted for about four weeks despite treatment with a penicillin antibiotic prescribed by one of your colleagues.

\section{Initial investigation and treatment}

- Organise an urgent chest X-ray (CXR) in view of the smoking history and persisting cough (see Box 1). CXR is widely and rapidly available, inexpensive and results in minimal radiation exposure.

- Send a sputum sample to microbiology for culture and antibiotic sensitivity testing.

- Consider a further course of antibiotic such as a macrolide to treat penicillin-resistant organisms and atypical organisms.
Box 2 Patients with a solitary lung shadow

\section{Relevant history}

- Respiratory

- Chest or shoulder pain

- Cough

Haemoptysis

Hoarseness of voice

Shortness of breath

Sputum production

Wheezing

- Constitutional

Lethargy

Night sweats

Poor appetite

Weight loss

- Asbestos exposure

- Previous malignancy

- Smoking history

- Travel history

Differential diagnoses

- Abscess

- Adenoma

- Aspergilloma

- Carcinoma

- Hamartoma

- Metastasis from another organ

- Pulmonary infarction

- Round atelectasis

- Round pneumonia

- Round pulmonary oedema

- Tuberculosis

\section{Positive CXR result}

The CXR shows a solitary mass-like shadow in the lung and is reported by the radiologist as being suspicious of lung cancer.

\section{Further history-taking}

- Revisit the history, paying particular attention to certain pertinent aspects that may point towards either lung cancer or other differential diagnoses (see Box 2).

- Discuss the benefits of stopping smoking. There is good evidence that smoking cessation improves outcome for patients with lung cancer. It reduces 
the perioperative risk and improves outcome following chemotherapy as well as improving long-term survival and quality of life in both small cell and non-small cell lung cancer (NSCLC).

\section{Examination findings, further investigation and referral}

- Examine the patient carefully, looking for finger clubbing and signs indicating locally extensive or metastatic disease, such as peripheral lymphadenopathy, organomegaly, superior vena cava (SVC) obstruction and stridor. Urgent referral for specialist care should be made whilst awaiting the results of CXR in patients who present with stridor or SVC obstruction.

- Assess the general fitness of the patient, taking into account the build of the patient and whether the patient appears well nourished or cachetic while making a note of the height and weight.

- Discuss the results of the CXR with the patient and convey your concerns regarding your suspicion of lung cancer if appropriate. Further tests will be required to clarify the diagnosis and extent of the problem. These will usually include a computed tomography (CT) scan of the thorax, and (depending on fitness) either bronchoscopy or percutaneous biopsy.

- Refer the patient to a chest physician without delay in keeping with the Department of Health guidelines on the '2-week wait' scheme for referral of patients with suspected lung cancer to secondary care.

- Address and treat any associated conditions

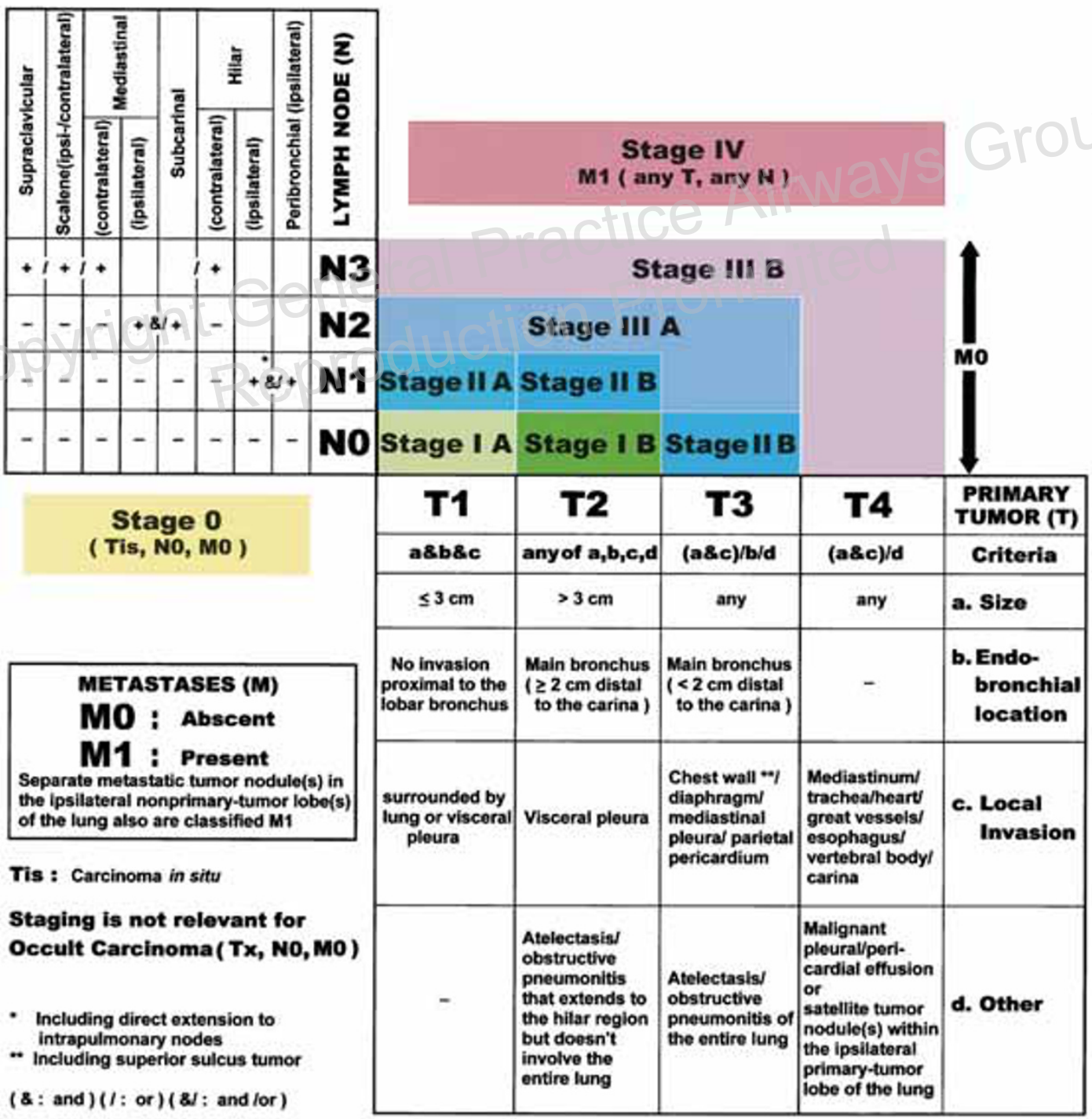

Table 1 Reference chart for TNM staging of lung cancer. Reproduced from Lababede et al. ${ }^{1}$ 
such as concomitant chest infection or airway bronchoconstriction for patient optimisation prior to further investigation and management.

\section{Staging classification}

\section{Non-small cell lung cancer}

NSCLC is the most common form of lung cancer, accounting for $80 \%$ of all lung cancers. It encompasses histological subtypes of adenocarcinoma, squamous cell carcinoma and large cell carcinoma. Although ultimately definitive treatment will vary with different subtypes of NSCLC, they are grouped together when staging lung cancer since they behave in a similar manner with respect to local or distant effects and in terms of potential tumour operability.

NSCLC is staged using the widely adopted TNM system as summarised in Table $1 .{ }^{1}$ It is based on tumour characteristics, nodal involvement of disease, and the extent of tumour spread beyond the primary lesion. When characterising a tumour, the size, location and local extent of the tumour is taken into account. Nodal stations are divided

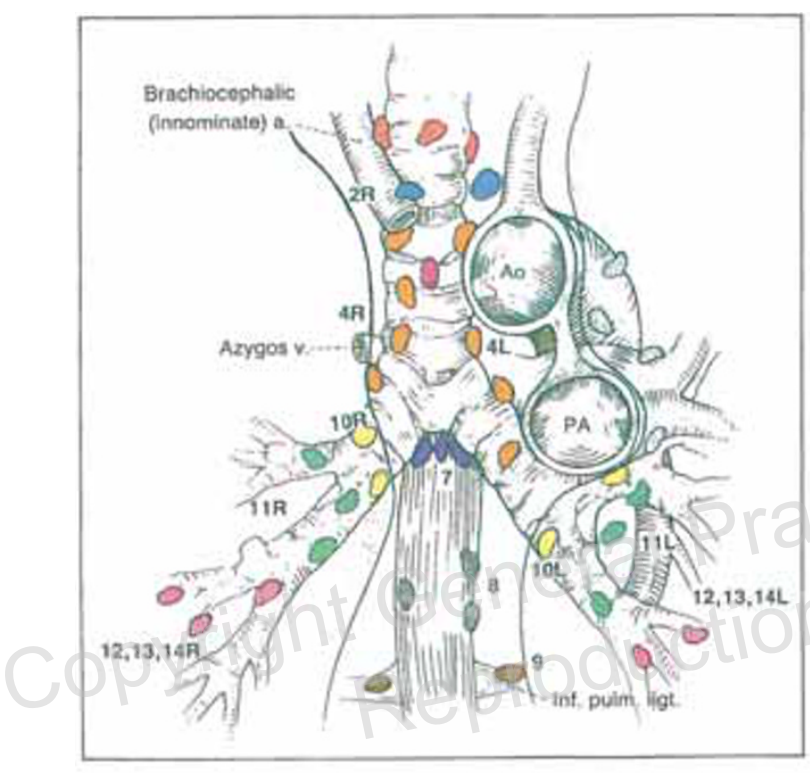
$\mathrm{N}_{2}$ NODES
SUPERIOR MEDIASTINAL NODES
1 Highest Mediastinal
2 Upper Paratracheal
3 Pre-vascular and Retrotracheal
- 4 Lower Paratracheal (including Azygos Nodes)

\section{AORTIC NODES \\ 5 Subaortic (A-P window) \\ 6 Para-aortic (ascending aorta or phrenic)}
INFERIOR MEDIASTINAL NODES
7 Subcarinal
8 Paraesophageal (below carina)
9 Pulmonary Ligament

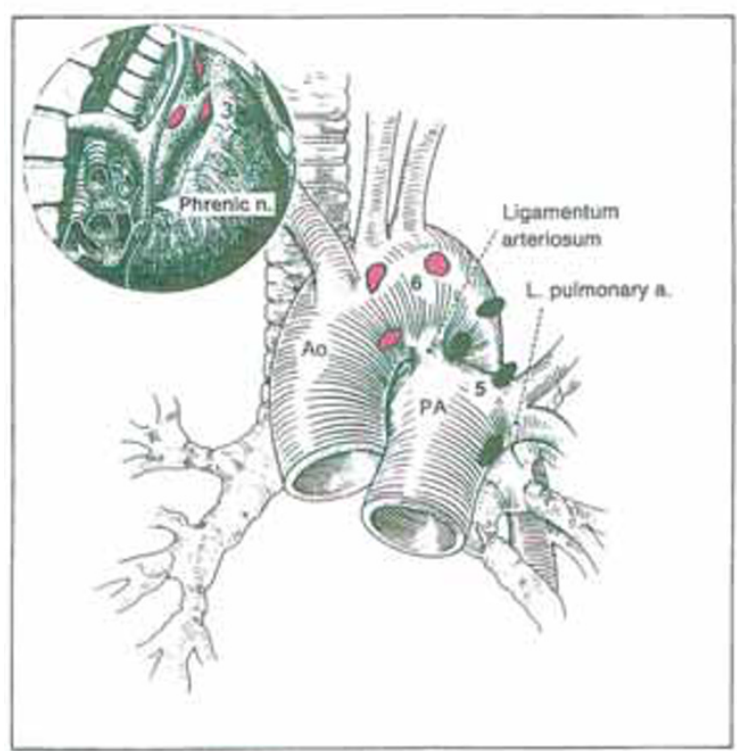

\begin{tabular}{l}
\hline$N_{1}$ NODES \\
\hline 10 Hilar \\
11 Interlobar \\
12 Lobar \\
13 Segmental \\
14 Subsegmental
\end{tabular}

Figure 1 Regional nodal stations for lung cancer staging. Reproduced from the American Thoracic Society and the European Respiratory Society. ${ }^{2}$ 
into different groups as depicted in Figure $1 .^{2}$ Patient symptoms and clinical signs will largely guide accurate determination and investigation of distant metastases. Patients with stage I - IIIA are considered potentially curable and are offered surgery following favourable work-up.

\section{Small cell lung cancer}

A separate staging system exists for small cell lung cancer in view of its inherently aggressive nature, with the majority of patients already suffering from metastatic disease at presentation. Classification is binomial comprising either limited or extensive disease.

\section{Traditional staging methods}

\section{Primary tumour}

\section{CT scanning}

CT scanning of the thorax is the most commonly used method to stage lung cancer and all patients will undergo this procedure. A CT scan is able to provide more detailed information compared to conventional chest radiography with regards to tumour characteristics, although CXR is by far the most common means by which initial suspicion of lung cancer is raised.

\section{Bronchoscopy}

Bronchoscopy is mainly used to investigate cases of haemoptysis in the hope of finding an endobronchial lesion to explain the presenting complaint. It is also a useful means of obtaining a tissue diagnosis should a tumour be seen in the bronchial tree. With regards to staging, the role of bronchoscopy is to ascertain the location of tumour in relation to its distance from the main carina in order to determine tumour operability.

\section{Mediastinal lymph nodes}

\section{CT scanning}

CT scanning will provide information with regards to the presence of abnormal enlarged lymph nodes and hence an indication of possible tumour involvement. However, CT scanning is not able to differentiate between tumour infiltration or inflammatory reaction as the cause of lymph node enlargement. Therefore, other methods such as fluorine-18 fluorodeoxyglucose positron emission tomography (PET) are required which can be used in conjunction with, or in parallel to, CT scanning in order to provide complementary information. A
Table 2 A comparison of sensitivities and specificities observed in mediastinal staging

\begin{tabular}{lcc}
\hline & Sensitivity (\%) & Specificity (\%) \\
\hline CT $^{2,3,6}$ & $64-86$ & $62-78$ \\
PET $^{6,7}$ & $73-94$ & $59-94$ \\
CT + PET (parallel) & 97 & 44 \\
PET/CT (integrated) & 85 & 84 \\
EBUS $^{4}$ & 85 & 100 \\
EUS $^{5}$ & 93 & 100 \\
\hline
\end{tabular}

summary of sensitivities and specificities of various methods used in mediastinal staging are given in Table 2.

\section{PET scanning}

PET is able to detect lymph nodes that are abnormal in terms of heightened metabolic activity and is useful in detecting tumour involvement of nodal tissue especially when correlated with other investigations. However, a positive PET result does not always necessarily suggest tumour involvement, as PET can be positive in non-malignant conditions such as granulomatous diseases and inflammatory processes.

Mediastinoscopy, anterior mediastinotomy and video assisted thoracic surgery (VATS)

Surgical techniques to obtain histopathological samples remain the gold standard and provide the highest order of lung cancer staging. However, not all patients are able to undergo such procedures especially if they have significant comorbidities that may put them at increased risk of perioperative complications.

\section{Metastasis}

\section{CT scanning}

CT is able to detect metastasis to other organs such as the brain, liver and adrenals. It is also able to pick up any gross abnormalities of bony tissues infiltrated by tumour.

\section{PET}

PET may be used to detect metastasis in soft tissue organs and bones.

\section{Bone scan}

Bone scan is performed as a complementary investigation for bone pain particularly when 
there are clinical symptoms or blood biomarkers to suggest bony metastasis.

\section{Novel staging methods}

\section{PET-CT}

In order to improve diagnostic accuracy, systems that combine the two separate modalities of CT and PET scanning have been developed. The so-called integrated PET-CT systems permit the coregistration of CT and PET images automatically while generating a more detailed data output. PET-CT has been shown to be significantly better in staging preoperative NSCLC as compared to CT alone. ${ }^{3}$ In the overall staging of primary tumour and nodal stations, PET-CT correctly staged lung cancer in $87 \%$ of patients compared to $66 \%$ with CT alone. The specificity and accuracy of PET-CT in malignant node depiction was also significantly better compared to CT alone ( $84 \%$ versus $69 \%$ respectively).

\section{Endobronchial ultrasound (EBUS) and endoscopic ultrasound (EUS)}

There is an unmet need in terms of minimally invasive procedures available for tissue sampling in the staging of mediastinal lymph nodes. EBUS and EUS have been developed to provide a comprehensive staging of the mediastinum through real-time ultrasound-guided fine-needle aspiration biopsy. EBUS is able to assess lymph nodes in stations 2, 3, 4, 5, 7, 10 and 11, and EUS in stations $4 \mathrm{~L}, 5,7,8$ and 9 . The sensitivity, specificity and accuracy of EBUS has been shown to be $85 \%, 100 \%$ and $89 \%$ respectively ${ }^{4}$ with the corresponding values for EUS of $93 \%, 100 \%$ and $95 \%$ respectively. ${ }^{5}$

\section{The future}

Technological advancement will continue to provide the backdrop for the development of more refined techniques and minimally invasive procedures in the staging of lung cancer. The former will allow for the detection of microinvasive disease whereas the latter will obviate the need for surgery. It is envisaged that the different modalities used in lung cancer staging will complement rather than supersede one another with benefit derived from a combination of methods rather than just one.

\section{Conflict of interest declaration}

The author has no conflicts of interest to declare.

\section{References}

1. Lababede 0 , Meziane MA, Rice TW. TNM staging of lung cancer: a quick reference chart. Chest 1999;115:233-5.

2. The American Thoracic Society and the European Respiratory Society. Pretreatment evaluation of nonsmall-cell lung cancer. Am J Respir Crit Care Med 1997; 156:320-32.

3. Shim SS, Lee KS, Kim BT et al. Non-small cell lung cancer: prospective comparison of integrated FDG PET/CT and CT alone for preoperative staging. Radiology 2005;236:101119.

4. Rintoul RC, Skwarski KM, Murchison JT, Wallace WA, Walker WS, Penman ID. Endobronchial and endoscopic ultrasound-guided real-time fine-needle aspiration for mediastinal staging. Eur Respir J 2005;25:416-21.

5. Caddy G, Conron M, Wright G, Desmond P, Hart D, Chen RY. The accuracy of EUS-FNA in assessing mediastinal lymphadenopathy and staging patients with NSCLC. Eur Respir J 2005;25:410-15.

6. Pozo-Rodriguez F, Martin de Nicolas JL, Sanchez-Nistal MA et al. Accuracy of helical computed tomography and [18F] fluorodeoxyglucose positron emission tomography for identifying lymph node mediastinal metastases in potentially resectable non-small-cell lung cancer. J Clin Oncol 2005;23:8348-56.

7. Weng E, Tran L, Rege S et al. Accuracy and clinical impact of mediastinal lymph node staging with FDG-PET imaging in potentially resectable lung cancer. $\mathrm{Am} \mathrm{J} \mathrm{Clin} \mathrm{Oncol}$ 2000;23:47-52.

\section{Useful reading}

Management of patients with lung cancer: a national clinical guideline. Scottish Intercollegiate Guidelines Network 2005. Available at: http://www.sign.ac.uk/pdf/sign80.pdf

Lung cancer: the diagnosis and treatment of lung cancer. National Institute for Clinical Excellence 2005. Available at: http: //www.nice.org.uk/pdf/CG024niceguideline.pdf

Lung cancer information centre. CancerBACUP 2005. Available at: http: //www.cancerbacup.org.uk/cancertype/lug

Lung cancer online. Lung Cancer Online Foundation 2005. Available at: http://www.lungcanceronline.org

Available online at http://www.thepcrj.org 